\title{
Solving the Triangular Ising Antiferromagnet by Simple Mean Field
}

\author{
Serge Galam ${ }^{1}$ and Pierre-Vincent Koseleff ${ }^{2}$ \\ ${ }^{1}$ Laboratoire des Milieux Désordonnés et Hétérogènes ${ }^{*}$ Case 86, \\ Université Pierre et Marie Curie, 4, place Jussieu, F-75252 Paris Cedex 05. \\ ${ }^{2}$ Équipe "Analyse Algébrique", Institut de Mathématiques † Case 82 \\ Université Pierre et Marie Curie, 4, place Jussieu, F-75252 Paris Cedex 05.
}

February 20, 2001

\begin{abstract}
Few years ago, application of the mean field Bethe scheme on a given system was shown to produce a systematic change of the system intrinsic symmetry. For instance, once applied on a ferromagnet, individual spins are no more equivalent. Accordingly a new loopwise mean field theory was designed to both go beyond the one site Weiss approach and yet preserve the initial Hamitonian symmetry. This loopwise scheme is applied here to solve the Triangular Antiferromagnetic Ising model. It is found to yield Wannier's exact result of no ordering at non-zero temperature. No adjustable parameter is used. Simultaneously a non-zero critical temperature is obtained for the Triangular Ising Ferromagnet. This simple mean field scheme opens a new way to tackle random systems.
\end{abstract}

${ }^{*}$ Laboratoire associé au CNRS (UMR n 7603), galam@ccr.jussieu.fr

${ }^{\dagger}$ Laboratoire associé au CNRS (UMR n 7586), koseleff@math.jussieu.fr 


\section{Introduction}

Collective phenomenon are rather difficult to solve exactly. Up to date, only some one dimensional problems and the square zero field Ising model allow an exact analytical solution [1]. To compensate this situation, a rich family of approximate methods has been developed over the last one hundred years. The most powerful one being the renormalization group techniques [2].

At start was the Mean Field Theory (MFT). It offers a very practical and simple tool to solve most collective phenomena [1]. While it is completely universal and generic, associated quantitative results are unusually poor. In particular critical temperatures and exponents are rather far from exact estimates [2]. Sometimes even the order of the transition may be wrong like for the instance in the Potts model [3].

The crudest and most simple version of MFT is the 1907 Weiss pioneer model [4]. It reduces the infinite number of fluctuating degrees of freedom down to one, $S_{i}$, which couples to homogeneous mean field degrees of freedom $m$. The thermodynamics is then solved calculating the associated partition function from which the self-consistent equation $\left.<S_{i}\right\rangle=m$ (where $\langle\ldots>$ means thermal average) is derived.

In the case of Ising systems with $q$ nearest neighbor interactions, Weiss theory gives $<S_{i}>=\tanh (K q m)$ where $K \equiv \beta J, J$ is the exchange coupling, $\beta \equiv \frac{1}{k_{B} T}, k_{B}$ is the Boltzman constant and $T$ is the temperature. Associated critical temperature is $K_{c}=\frac{1}{q}$. At odd with the known exact result a phase transition is obtained at $d=1(q=2)[1]$.

From there it took 28 years before Bethe improved the Weiss model [5]. Instead of just one fluctuating spin, he considers a cluster of fluctuating spins with a central one and its nearest neighbors. The main achievement of the Bethe approximation is to yield the exact result of no ordering at one dimension. However, critical temperatures given by $K_{c}=\tanh ^{-1}\left(\frac{1}{q-1}\right)$, are not much better than from Weiss model. Critical exponents stay unchanged. Latter on, using computer capabilities, larger size fluctuating clusters have been considered to obtain better critical temperatures [6].

However, few years ago the Bethe cluster scheme was showed to systematically change the system intrinsic symmetry [7]. Starting from a system with equivalent sites like for instance a square Ising Ferromagnet, It ends up making individual sites unequivalent. At this stage it is worth to stress that an approximation can be very crude and yet not wrong as long as it preserves the intrinsic symmetry of the problem. Otherwise it does change the physics of it. We are not talking here about a symmetry breaking of the higher phase symmetry as it occurs in a usual phase transition but of a change of the symmetry of the disorder phase itself.

On this basis the challenge was to find out if it is indeed possible to build a MFT which considers more than two fluctuating spins, yet preserving the initial lattice symmetry. Indeed, Galam showed it is possible using a loopwise scheme (LWS) which articulates on finite-size one-dimensional closed loops [7]. Paving the whole lattice with these loops, half of them are kept fluctuating while the other half is averaged out with mean field degrees of freedom. The scheme is illustrated in Figure 1 for the square lattice.

The LWS is a generic model. It was applied to a large class of ferromagnetic systems 
Figure 1: The loopwise scheme in the square case: s1, s2, s3, s4 are the fluctuating spins while $\mathrm{m} 1, \mathrm{~m} 2, \mathrm{~m} 3, \mathrm{~m} 4$ are mean field averages

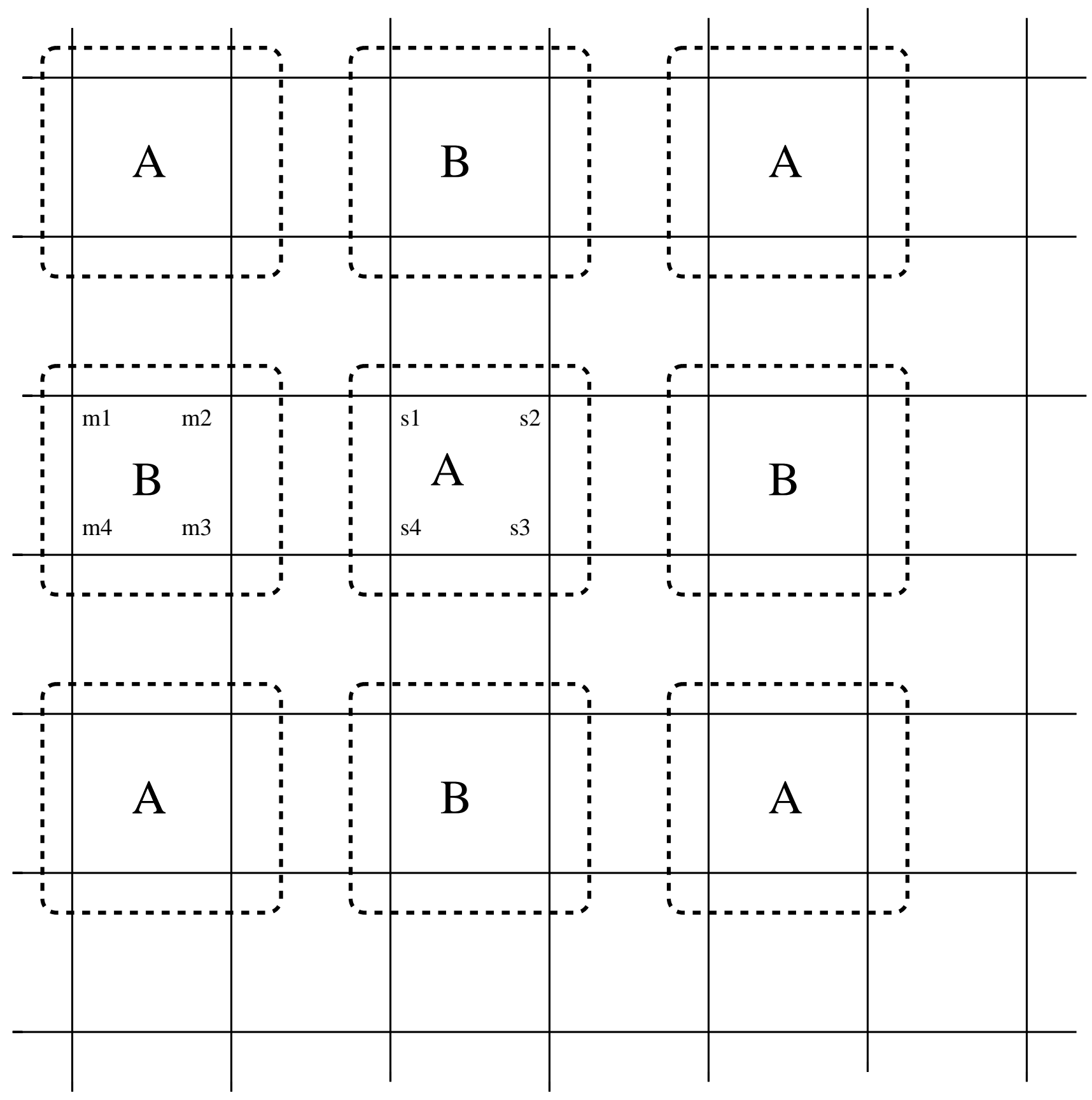


on Bravais lattices $[7,8]$. It reproduces the exact result of no ordering at one dimension. Moreover, for Ising hypercubes, it exhibits a lower critical dimension $d_{l}$ for long range ordering which is equal to the Golden number $d_{l}=\frac{1+\sqrt{5}}{2}$. However critical exponents are unchanged from Weiss model.

On this basic, to determine the range of validity of this new LWS, it is of interest to check if it can yield new properties which are out of reach of previous mean field theories like frustration. For instance, when applied to the fully-frustrated Triangular Ising Antiferromagnet (TIA) most MFT predict a transition at a non-zero temperature while an earlier exact argument by Wannier proved no symmetry breaking occurs at any non-zero temperature [9].

Few years ago, to bridge this difficulty Netz and Berker introduced the hard spin recipe [10]. It combines a mean field calculation with some Monte Carlo sampling. When applied to the TIA, it yields the correct result of no ordering at $T \neq 0$. Later Banavar et al suggested that the Monte Carlo sampling could be reproduced by expanding all possible products of the 6 nearest neighbors spins of the "exact spin" but it was then disproved by Netz and Berker[11].

More recently focusing on the TIA, Monroe approximated the triangular lattice with a Husimi tree built up of triangles [12]. It then allows to include properly frustration to get a correct phase diagram. However an Huzimi tree is not a triangular lattice.

In this paper we apply the very simple LWS to the fully frustrated Triangular Ising Antiferromagnet (TIA). The Wannier exact result is recovered [10] and a transition is found at $T=0$. The following of the paper is organized as follows. Section 2 deals with the frustration effect. In Section 3 the LWS is presented. The TIA is solved analytically in Section 4 using the LWS. In Section 5 using the same Equations, the Triangular Ising Ferromagnet (TIF) is also solved. Some possible applications are mentinned in the Last Section.

\section{The Frustration effect}

Frustration is a major ingredient of many physical systems. It results from the impossibility to minimize simultaneously all pair interactions. In turn it makes the ground state highly degenerate [9]. Frustration effects may arise from either quenched disorder or topological constraints.

Random bond spin glasses are the archetype of frustration produced by disorder. The random distribution of quenched competing interactions generates analytical difficulties in calculating the thermodynamic functions. In particular to average the disorder over the logarithm of the partition function is yet a real theoretical challenge. Usual mean field treatments failed to incorporate simultaneously frustration and quenched randomness.

On this basis the TIA has the advantage of being fully frustrated without any disorder making the study of frustration itself more easy. It is therefore the perfect candidate to check the ability of a new scheme to deal with frustration. In addition an earlier exact argument by Wannier [10] has proved the absence of symmetry breaking at any non- 
zero temperature for this system. At constrast most mean field like approaches produce wrongly some non-zero critical temperature. Along this line, Netz and Berker recipe [10] with Banavar et al reformulation [11] stand at odd.

\section{The loopwise scheme (LWS)}

The LWS was introduced few years ago to overpass the symmetry inconsistency of the Bethe scheme, yet retaining its physical feature of including several fluctuating degrees of freedom [7].

To implement the LWS on any lattice requires to single out two identical interpenetrating sublattices. Each element being composed from a closed compact loop of degrees of freedom. The shape and number of these degrees of freedom are determined by the lattice topology. It is the smallest closed linear loop. For instance in the square case (Fig. 1) it includes 4 spins while for the triangular lattice (Fig. 2) 3 spins are involved. One of the sublattice is fluctuating and the other one is mean field.

Both sublattices are coupled via nearest neighbor interactions. The problem is thus mapped onto decoupled one-dimensional closed fluctuating chains in external fields. The fields originate from the coupling to the mean field loops. At this stage an exact analytical

calculation can be performed whatever the chain size is. It is worth to note no adjustable parameter is used.

The LWS is a generic model. It was applied to a large class of ferromagnetic systems $[7,8]$. Being built on using closed linear loops it should be well adapted to embody frustration effects [9].

\section{Solving the Triangular Ising Antiferromagnet}

We now apply the LWS to the fully frustrated TIA. We first partition the triangular lattice into two interpenetrated triangular sublattices $A$ and $B$. Thermal fluctuations are then ignored on the B-sublattice while preserved within the A-sublattice. These triangles are closed loops with no center (see Figure 2).

All nearest neighbor (nn) plaquettes of a A-plaquette are B plaquettes, and vice versa. Therefore, on a given plaquette each spin has two nn spins of the same species (within the same plaquette), and four nn spins of the other species (belong respectively to three different nn plaquettes).

Above breaking of the initial lattice symmetry makes the partition function calculable by decoupling the fluctuating triangles. The A-sublattice degrees of freedom can thus be integrated out in the partition function. The initial lattice symmetry will be restored latter using the usual mean field self-consistent constraint (Eq. (田) below). 
Figure 2: The loopwise scheme in the triangular case: s1, s2, s3 are the fluctuating spins while 1, 2, 3 represent mean field averages $\mathrm{m} 1, \mathrm{~m} 2, \mathrm{~m} 3$

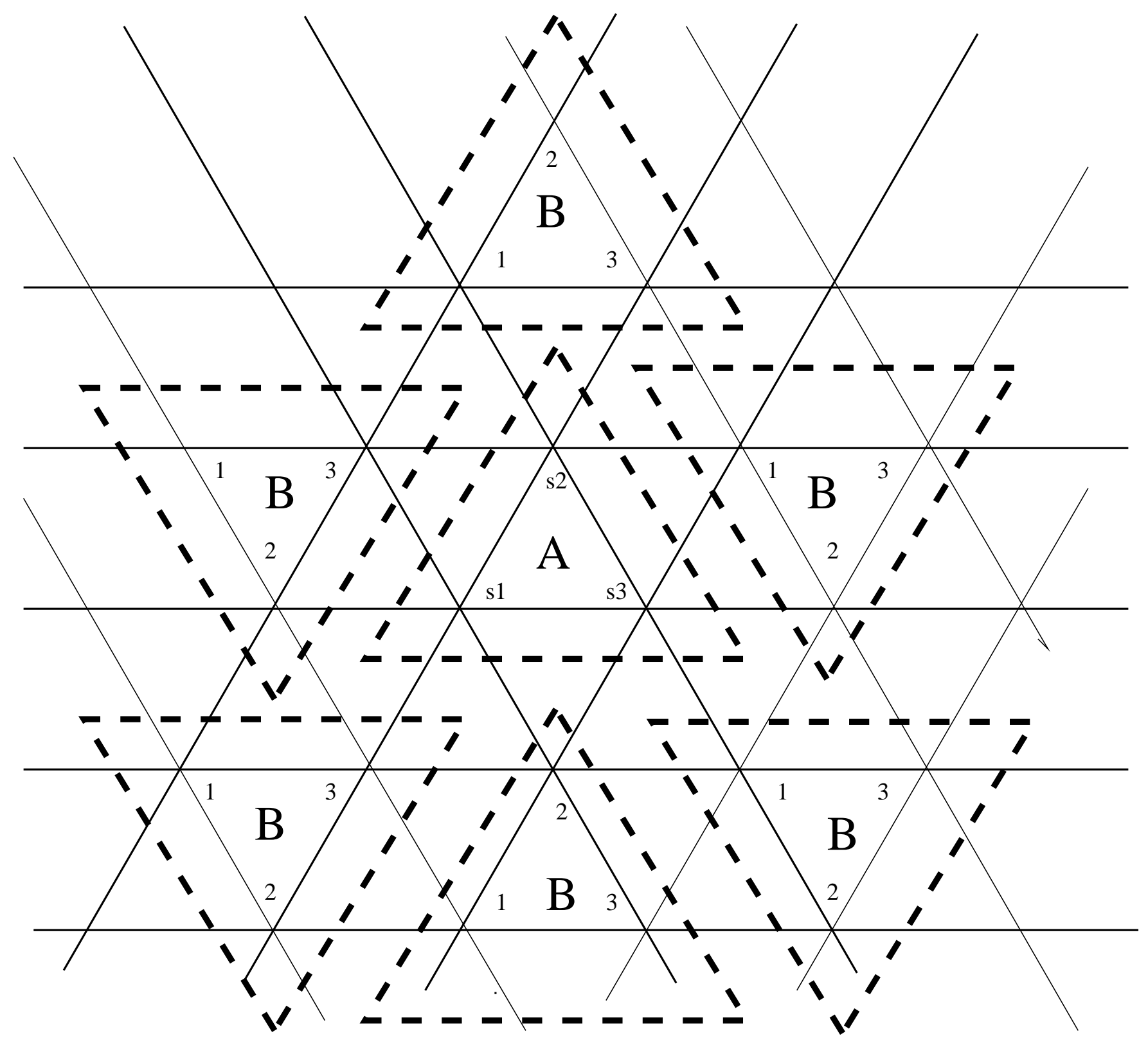




\subsection{Setting the Equations}

Given a A plaquette, we label the 3 fluctuating spins $S_{1}, S_{2}, S_{3}$. We then introduce 3 magnetizations $m_{1}, m_{2}, m_{3}$ for corresponding B plaquettes (Fig. 3). The Hamiltonian then writes,

$$
H=-J\left(S_{1} S_{2}+S_{2} S_{3}+S_{3} S_{1}\right)-\delta J\left(S_{1}\left(m_{2}+m_{3}\right)+S_{2}\left(m_{3}+m_{1}\right)+S_{3}\left(m_{1}+m_{2}\right)\right),
$$

where $\delta=2$ accounts for the coupling to the B mean field plaquettes. From Eq. (11) the partition function is

$$
Z=\sum_{S_{i}= \pm 1} \exp \{-\beta H\}
$$

where $i=1,2,3$. The three thermal average of $S_{1}, S_{2}, S_{3}$ are given by,

$$
<S_{i}>=\frac{1}{Z} \sum_{S_{j}= \pm 1} S_{i} \exp \{-\beta H\} .
$$

We can thus write the associated three self-consistent equations,

$$
<S_{i}>=m_{i}
$$

\subsection{Looking for minima}

Indeed we are looking for minina of the free-energy which results from the partition function $Z$. It is then worth to stress not all solutions of Eq. (1) are minimum. A criterium to make Eq. (4) a derivative of a function is to require its cross derivatives with respect to the $m_{i}$ to be equal, i. e.,

$$
\frac{\partial}{\partial m_{j}}<S_{i}>=\frac{\partial}{\partial m_{i}}<S_{j}>,
$$

for $i, j=1,2,3$

Writing, $\mathbf{S}=\left(<S_{1}>,<S_{2}>,<S_{3}>\right)$, and $\mathbf{m}=\left(m_{1}, m_{2}, m_{3}\right)$, the problem is now to find a set $S=\left\{\mathbf{m} \in \mathbf{R}^{3} ; \mathbf{S}(\mathbf{m})=\mathbf{m}\right\}$, such that there exists a function $F$ obeying to,

$$
(\mathbf{m}-\mathbf{S}(\mathbf{m}))=\mathrm{d} F(\mathbf{m})=0 .
$$

To solve it, we rewrite thermal averages $<S_{i}>$ as,

$$
<S_{i}>=\frac{1}{Z} \sum_{s_{i}= \pm 1} s_{i} f\left(s_{1}, s_{2}, s_{3}\right)
$$

where

$f\left(s_{1}, s_{2}, s_{3}\right)=\exp \left\{K\left(s_{1} s_{2}+s_{1} s_{3}+s_{2} s_{3}\right)+\delta K\left(m_{1}\left(s_{2}+s_{3}\right)+m_{2}\left(s_{1}+s_{3}\right)+m_{3}\left(s_{1}+s_{2}\right)\right)\right\}$. 
Let $\sigma \in \Sigma_{3}$ be a permutation. Considering $\sigma(\mathbf{m})=\left(m_{\sigma(1)}, m_{\sigma(2)}, m_{\sigma(3)}\right)$ we have,

$$
\sigma(\mathbf{Z}(\mathbf{m}))=\mathbf{Z}(\sigma(\mathbf{m})), \mathbf{Z}(-\mathbf{m})=-\mathbf{Z}(\mathbf{m}) .
$$

Writing $X=\exp K$ and $x_{i}=\exp \delta K m_{i},<S_{i}>$ are rational fractions in $\left(x_{i}, X\right)$ and we have,

$$
\begin{gathered}
Z=\frac{D}{X T_{3}^{2}}, \\
D=\left(1+T_{3}^{2}\right) X^{4}+T_{2}+T_{1} T_{3}, \\
<S_{i}>=1-2 \frac{x_{i}\left(T_{1}+T_{3}-x_{i}\right)+X^{4}}{D},
\end{gathered}
$$

where

$$
\begin{array}{r}
T_{1}=x_{1}+x_{2}+x_{3}, \\
T_{2}=x_{1} x_{2}+x_{1} x_{3}+x_{2} x_{3}, \\
T_{3}=x_{1} x_{2} x_{3},
\end{array}
$$

are the elementary symetric functions. Note $D>0, X>0, x_{i}>0$ and $\left|<S_{i}>\right|<1$.

Solving first the $K=0$ case, we get immediately $\left\langle S_{i}\right\rangle=0$ and the solution is $m_{i}=0$. We can then proceed assuming $K \neq 0$.

\subsection{The most general solution $m_{1} \neq m_{2} \neq m_{3}$}

We can now solve the equations, starting with the most general case $m_{1} \neq m_{2} \neq m_{3}$. Eq. (5) is equivalent to,

$$
\frac{\partial<S_{i}>}{\partial x_{j}} \frac{\partial x_{j}}{\partial m_{j}}=\frac{\partial<S_{j}>}{\partial x_{i}} \frac{\partial x_{i}}{\partial m_{i}}
$$

that is,

$$
\begin{aligned}
& \left(x_{1}-x_{2}\right)\left(X^{4} x_{3}^{2} x_{2}^{3} x_{1}^{3}-x_{1}^{2} x_{2}^{2}+2 X^{4} x_{1}^{2} x_{2}^{2} x_{3}^{2}+x_{3}^{2} x_{2} x_{1}-2 X^{4} x_{1} x_{2}-X^{4}\right)=0 \\
& \left(x_{1}-x_{3}\right)\left(X^{4} x_{2}^{2} x_{3}^{3} x_{1}^{3}-x_{3}^{2} x_{1}^{2}+2 X^{4} x_{1}^{2} x_{2}^{2} x_{3}^{2}+x_{2}^{2} x_{3} x_{1}-2 X^{4} x_{3} x_{1}-X^{4}\right)=0 \\
& \left(x_{2}-x_{3}\right)\left(X^{4} x_{1}^{2} x_{2}^{3} x_{3}^{3}-x_{2}^{2} x_{3}^{2}+2 X^{4} x_{1}^{2} x_{2}^{2} x_{3}^{2}+x_{1}^{2} x_{3} x_{2}-2 X^{4} x_{3} x_{2}-X^{4}\right)=0 .
\end{aligned}
$$

Suppose first, three different values of $m_{i}$. It makes $x_{1} \neq x_{2} \neq x_{3}$ since $K \neq 0$, which in turn, solving Eq. (15) implies,

$$
T_{1}=-\frac{T_{2}}{T_{3}} \frac{2 T_{3}^{2}-1}{T_{3}^{2}-2},
$$


and,

$$
X^{4}=\frac{T_{2}}{T_{3}^{2}-2} .
$$

In conclusion

$$
D=\left(1+T_{3}^{2}\right) X^{4}+T_{2}+T_{1} T_{3},=0
$$

which is impossible since $D>0$. Therefore, we can conclude that out of the three $m_{i}$, two must be equal. We then suppose $m_{1}=m_{2} \neq m_{3}$.

\subsection{The solution exhibits the symmetry $m_{1}=m_{2} \neq m_{3}$}

From above calculation we restrict the minima search to the subspace of solution $m_{1}=$ $m_{2} \neq m_{3}$. It implies $x_{1}=x_{2}$ and,

$$
<S_{1}>-<S_{3}>=-2 \frac{x_{2}\left(1+x_{1} x_{3}\right)}{D}\left(x_{1}-x_{3}\right)
$$

so it makes,

$$
\frac{\exp \left(\delta K m_{1}\right)-\exp \left(\delta K m_{3}\right)}{m_{1}-m_{3}}<0,
$$

which in turn makes $K<0$. Let us define $P \equiv x_{2} x_{3}$ and $N \equiv x_{2}^{2} x_{3}$, it gives,

$$
X^{4}=\frac{P^{3}-N^{2}}{P\left(N^{2} P+2 N^{2}-2 P-1\right)},
$$

and,

$$
<S_{1}>+<S_{2}>+<S_{3}>=\frac{P-1}{P+1}
$$

If $P=1$ or $N=1$ then $m_{1}=m_{2}=m_{3}=0$ which is not possible since we assumed above $m_{1}=m_{2} \neq m_{3}$. So $(P-1)(N-1) \neq 0$. On the other hand, as $K<0$, we must have,

$$
\frac{1-N}{m_{1}+m_{2}+m_{3}}>0
$$

which makes $(P-1)(N-1)<0$ but Eq. (21) gives,

$$
X^{4}=\frac{1}{P} \frac{P^{3}-N^{2}}{(1-P)+(2+P)\left(N^{2}-1\right)}<0,
$$

which is impossible. In conclusion all three $m_{i}$ must be equal. On this basis we now assume $m_{1}=m_{2}=m_{3}=m$. 


\subsection{The solution is fully symmetrical with $m_{1}=m_{2}=m_{3}=m$}

We have now proved the minima belong to the solution subspace defined by the symmetry

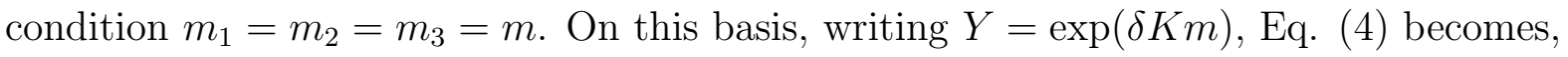

$$
m=f_{K}(m)=\frac{\left(Y^{4}-1\right)\left(\left(Y^{8}+Y^{4}+1\right) X^{4}+Y^{4}\right)}{\left(Y^{4}+1\right)\left(\left(Y^{8}-Y^{4}+1\right) X^{4}+3 Y^{4}\right)},
$$

so we have either $m=0$, or both,

$$
\frac{Y^{4}-1}{m}=\frac{e^{4 \delta K m}-1}{m}>0,
$$

and $K>0$. We also deduce that $\left|f_{K}(m)\right|<1$.

As $f_{K}(-m)=-f_{K}(m)$ it is enough to solve the case $m \geq 0$. We thus obtain $X>$ $1, Y>1, K>0$, or $m=0$. Computing the derivative gives,

$$
\begin{aligned}
& f_{K}^{\prime}(m)=8 \delta K \frac{Y^{4}\left(3 Y^{8} X^{8}+\left(Y^{16}+4 Y^{12}+4 Y^{4}+1\right) X^{4}+3 Y^{8}\right)}{\left(Y^{4}+1\right)^{2}\left(\left(Y^{8}-Y^{4}+1\right) X^{4}+3 Y^{4}\right)^{2}}, \\
& f_{K}^{\prime \prime}(m)=-32 \delta^{2} K^{2} \frac{Y^{4}\left(Y^{4}-1\right)}{\left(Y^{4}+1\right)^{3}\left(\left(Y^{8}-Y^{4}+1\right) X^{4}+3 Y^{4}\right)^{3}} g_{K}(m),
\end{aligned}
$$

where

$$
\begin{aligned}
g_{K}(m)= & \left(Y^{4}+1\right)^{6}+\left(2 Y^{8}+7 Y^{4}+2\right)\left(Y^{4}+1\right)^{4}\left(X^{4}-1\right) \\
& +\left(Y^{16}+7 Y^{12}+21 Y^{8}+7 Y^{4}+1\right)\left(Y^{4}+1\right)^{2}\left(X^{4}-1\right)^{2} \\
& +9 Y^{8}\left(Y^{8}+Y^{4}+1\right)\left(X^{4}-1\right)^{3}>0 .
\end{aligned}
$$

Therefore when $m \geq 0$, in addition to $0 \leq f_{K}(m)<1$, we have $f_{K}^{\prime}(m)>0$ and $f_{K}^{\prime \prime}(m)<0$. These properties allow to conclude that,

1. If $f_{K}^{\prime}(0) \leq 1,0$ is the only fixed point of $f_{K}$.

2. If $f_{K}^{\prime}(0)>1, f_{K}$ has exactly three fixed points, $0, b,-b$ where $-1<b<1$

Computing then,

$$
f_{K}^{\prime}(0)=2 \delta K \frac{3 \exp (4 K)+1}{\exp (4 K)+3},
$$

it appears to be an increasing function of $K$. It makes,

$$
f_{K}^{\prime}(0)=1
$$

to have a unique solution $K_{0}$. Moreover, if $K<K_{0}$ then $f_{K}^{\prime}(0)<1$ and if $K>K_{0}$ then $f_{K}^{\prime}(0)>1$. 


\subsection{The actual minima}

Looking for minima of $F_{K}$ where,

$$
\frac{\mathrm{d} F_{K}(m)}{\mathrm{d} m}=m-f_{K}(m)
$$

depending on the value of $K$, two cases appear quite naturally for $K>K_{0}$ and $K \leq K_{0}$. It shows the Triangular Ising both Anti and Ferromagnets are solved simultaneously.

\subsubsection{First case: $K>K_{0}$}

In this case, $f_{K}(m)=m$ has 2 solutions $m=0$ and $m^{2}=a$ where $a$ is a positive function of $K$. Having,

$$
F_{K}^{\prime \prime}(m=0)=1-f_{K}^{\prime}(m=0)<0
$$

$m=0$ is a maximum for $F_{K}$. In parallel,

$$
F_{K}^{\prime \prime}(m=\sqrt{a})=F_{K}^{\prime \prime}(m=-\sqrt{a})=1-f_{K}^{\prime}\left(m_{1}\right)>0 .
$$

Therefore $m=\sqrt{a}$ and $m=-\sqrt{a}$ are minima of $F_{K}$. They correspond to the Triangular Ising Ferromagnet symmetry breaking at low temperatures where $K_{0}$ is the associated critical temperature.

\subsubsection{Second case: $K \leq K_{0}$}

Then the unique solution of $f_{K}(m)=m$ is $m=0$. There,

$$
F_{K}^{\prime \prime}(0)=1-f_{K}^{\prime}(0)>0,
$$

so it is a minimum for $F_{K}$. This case embodies indeed two different physical situations.

1. The first range of positive $K, 0 \leq K \leq K_{0}$, corresponds to the disordered phase of above Triangular Ising Ferromagnet.

2. At the same time, the range of negative $K(K \leq 0)$ corresponds to the Triangular Ising Antiferromagnet. For this system the unique solution is always $m=0$ for the whole range of temperatures $T>0$. We conclude situation proving thus no It means no ordering occurs for the TIA at any non zero temperature. The Wannier argument is thus recovered [10].

\subsection{A transition at $T=0$}

From the exact Wannier solution the Triangular Ising Antiferromagnet is known to exhibit a phase transition at $T=0$ to an ordered phase with broken symmetry among the three sublattices. Accordingly we now examine what our scheme yield in the case $K \rightarrow \infty$. To 
solve the equations it is more convenient to rewrite $Z$ and $<S_{i}>$ in terms of $T=\tanh (K)$ and $t_{i}=\tanh \left(\delta K m_{i}\right)$. We first note $\left|<S_{i}>\right| \leq 1$ since $|\sinh (x)| \leq \cosh (x)$. Then, once the $m_{i}$ are fixed within $[-1,1]$, the condition $K \rightarrow \infty$ make the $t_{i}$ to go to either one of the three values $-1,0,1$.

Computing $<S_{i}>$ in terms of $t_{i}$ and $K$ for each one of the 27 possible limit values of the $t_{i}$ set, we find 7 solutions for the $m_{i}$ which are respectively,

$$
m_{i}=0, i=1,2,3 \text {. }
$$

and

$$
m_{i}=m_{j}=-m_{k}= \pm 1 \text {. }
$$

To determine the actual minimum at $T=0$ we compute the associated values for free energy $F=-k_{B} T \log Z$. The first solution $m_{1}=m_{2}=m_{3}=0$ yields,

$$
F=-\frac{J}{K} \log ((6+2 \exp (4 K)) \exp (-K)) \underset{K \rightarrow-\infty}{\longrightarrow} 1
$$

and for $m_{1}=m_{2}=1, m_{3}=-1$ we get,

$$
F=-\frac{J}{K} \log (2 \exp (-K) \cos (2 \delta K)(3+\exp (4 K))) \underset{K \rightarrow-\infty}{\longrightarrow} 1-2 \delta
$$

making the solution $m_{1}=m_{2}=1, m_{3}=-1$ the minimum. However from Eqs. (38) and (39) the two free energies of the ordered/disordered phases are expected to become equal only at some non zero temperature, a little bit above zero temperature, that is quite close to a critical point. It is coherent to the known result of a phase transition for the Triangular Ising Antiferromagnet at $T=0$ in agreement with the previous improved mean field theory by Netz and Berker [10].

\section{The Triangular Ising Ferromagnet}

Coming back to the TIF, we can go further and evaluate the value of the critical temperature $K_{0}$. At this stage it is worth to notice, all above results are independent of the value of $\delta$ which accounts for the coupling to the mean field loops.

Since $1 \leq \frac{3 \exp (4 K)+1}{\exp (4 K)+3} \leq 3$, when $K>0$, from Eq. (31) we obtain,

$$
\frac{1}{6 \delta} \leq K_{0} \leq \frac{1}{2 \delta}
$$

In addition, in the limit of large $\delta$, we get,

$$
K_{0}=\frac{1}{2 \delta}-\frac{2}{\delta^{2}}+\frac{3}{4 \delta^{3}}-\frac{29}{24} \frac{1}{\delta^{4}}+\mathcal{O}\left(\frac{1}{\delta^{5}}\right) .
$$


To get a numerical estimate of the ferromagnetic critical temperature $K_{0}$ requires to have the $\delta$ value.

From Eq. (11) a straightforward arithmetic leads to $\delta=\frac{q-2}{2}=2$ since $2 \mathrm{nn}$ are treated exactly within the fluctuating loop out of the 6 triangular $\mathrm{n} n$. Plugging then, $\delta=2$ into Eq. (31) yields $K_{0}=0.1772$. It is rather far from the exact numerical estimate $K_{C}^{e}=0.2746$ [14]. In comparison, a usual mean field gives $K_{0}=\frac{1}{6}=0.1667$, while for Bethe it is $K_{0}=\tanh ^{-1}\left(\frac{1}{5}\right)=0.2027$.

\section{Conclusion}

In conclusion, we have showed that the very simple and generic mean field Loopwise Scheme, proposed by Galam [7], is able to solve exactly the Triangular Ising Antiferromagnet. Without any adjustable parameter it recovers the exact Wannier argument of no ordering at $T \neq 0$ and a transition at $T=0$ [10]. From the same Equations the Triangular Ising Ferromagnet is also solved simultaneously. A phase transition is obtained into a ferromagnetic phase at a non-zero critical temperature

Moreover, contrary to the Bethe scheme, it preserves the initial lattice symmetry, yet going beyond the one-site Weiss approach. It also yields no transition for Ising hypercubes at $d=1$ with a lower critical dimension of $d_{l}=\frac{1+\sqrt{5}}{2}$.

The Loopwise Scheme should allow a new solving of a very large class of physical systems, in particular random systems with frustration. For future work we consider to apply it first to the Triangular Ising Antiferromagnet in a finite field and then on the stacked 3D version of it. Application to the Random Field Ising model should also be done.

\section{Acknowledgments.}

We would like to thank Y. Shapir and R. Netz for stimulating discussion on the manuscript. 


\section{References}

1. R. K. Pathria, Statistical Mechanics, Pergamon Press (1972)

2. Sh-k Ma, Modern Theory of Critical Phenomena, The Benjamin Inc.: Reading MA (1976)

3. F. Y. Wu, Rev. Mod. Phys. $\underline{54}, 235$ (1982)

4. P. Weiss, J. Phys. Radium, Paris $\underline{6}, 667$ (1907)

5. H. A. Bethe, Proc. Roy. Soc. London A $\underline{150}, 552$ (1935)

6. M. Suzuki, Prog. Theor. Phys. $\underline{42}$ 1086-1097 (1969)

7. S. Galam, Phys. Rev. B $\underline{54}, 15991$ (1996)

8. S. Galam, J. Appl. Phys. B $\underline{87}, 7040$ (2000)

9. G. Toulouse, Commun. Phys. $\underline{2}, 115$ (1977)

10. G. H. Wannier, Phys. Rev. $\underline{79}, 357$ (1950)

11. R. R. Netz and A. N. Berker, Phys. Rev. Lett. $\underline{6}, 1377$ (1991)

12. J. R. Banavar, M. Cieplak, and A. Maritan, Phys. Rev. Lett. 67, 1807 (1991) and Reply by R. R. Netz and A. N. Berker

13. J. Monroe, Physica A $\underline{256}$, pages 217 (1998)

14. J. Adler, in "Recent developments in computer simulation studies in Condensed matter physics", VIII, edited by D. P. Landau, Springer (1995) 